\title{
Real-Time Demonstration of 28 Gbit/s Electrical Duobinary TDM-PON Extension Using Remote Nodes
}

\author{
R. Bonk ${ }^{1}$, R. Borkowski ${ }^{1}$, W. Poehlmann ${ }^{1}$, J. Van Kerrebrouck ${ }^{2}$, C. Chase ${ }^{3}$, R. Lucas ${ }^{3}$, T. De Keulenaer ${ }^{2}$, \\ J. Bauwelinck ${ }^{2}$, D. Van Veen ${ }^{4}$, V. Houtsma ${ }^{4}$, X. Yin ${ }^{2}$ and Th. Pfeiffer ${ }^{1}$ \\ 1:Nokia, Bell Labs, Lorenzstrasse 10, 70435 Stuttgart, Germany; e-mail: rene.bonk@nokia-bell-labs.com; \\ 2:Ghent University - imec, IDLab, Dep. INTEC, Technologiepark 15, 9052 Gent-Zwijnaarde, Belgium \\ 3:Bandwidth 10, 1218 Seventh St., Suite A, Berkeley, CA, 94710, USA \\ 4:Nokia, Bell Labs, 600-700 Mountain Avenue, Murray Hill, NJ, 07974, USA
}

\begin{abstract}
An experimental real-time reach and split extension of a $28 \mathrm{Gbit} / \mathrm{s}$ electrical duobinary TDM-PON is demonstrated. $50 \mathrm{~dB}$ budget is achieved using either remote nodes based on SOA or based on a distributed OLT concept.

OCIS codes: (060.0060) Fiber optics and optical communications, (060.4250) Networks.
\end{abstract}

\section{Introduction}

The optical access networks of multiple-system operators (MSO) cause challenges beyond the capabilities of traditional passive optical network (PON) solutions in terms of fiber reach and split ratio [1]. Many MSOs are constrained with fewer feeder fibers than required to support dense groups of subscribers. Hence, these operators require split ratios, e.g. 1:1024, which are much larger than typically supported by common PON solutions, e.g. 1:32. Additionally, particularly cable operators often have to bridge fiber distances exceeding $80 \mathrm{~km}$ to connect their head-ends (central office) with the customer premises. Therefore, the required fiber distance is also longer than conventionally supported by PON solutions, e.g. up to $20 \mathrm{~km}$. On top of this, many MSO attempt to consolidate and centralize their access network architectures to reduce cost and to simplify maintenance needs. This approach expands the mentioned specifications even further. Beside the network architecture challenges, operators are facing an ongoing bandwidth request from subscribers who are interested in e.g., 8K ultra-high definition video streaming or augmented reality applications. To satisfy the customer demands, an increase in line rate of the access network systems needs to be offered. Here, the next-generation of Ethernet-PON (100G-EPON) using $25 \mathrm{Gbit} / \mathrm{s} / \lambda$ [2, 3] is a potential candidate for future deployments. In a nutshell, MSOs need a way to support longer reach, higher split and higher bandwidth access networks utilizing a low-cost system technology. A promising solution to this deficiency can be the use of the low-cost $25 \mathrm{Gbit} / \mathrm{s}$ time-division multiplexed (TDM)-PON platform that is upgraded by a remote-PON node (RPN) to extend reach, minimize fibers required to serve subscribers, and allow for large centralized systems. Exemplarily, this RPN can be realized by an optical amplifier or by a distributed optical line terminal (OLT), i.e., extending the conventional PON by a local remote-PON network using a PON-to-PON concept.

In this paper, we experimentally demonstrate in real-time two solutions to satisfy MSOs access network demands for increased split, longer reach and higher line rates. A $28 \mathrm{Gbit} / \mathrm{s}$ electrical duobinary (EDB)-TDM-PON is extended by remote-PON nodes. A power budget exceeding $50 \mathrm{~dB}$ and up to $80 \mathrm{~km}$ fiber reach is achieved using either a node based on semiconductor optical amplifier (SOA) or based on a distributed OLT disaggregating the $28 \mathrm{Gbit} / \mathrm{s}$ EDB-TDM-PON to a $7 \mathrm{Gbit} / \mathrm{s}$ on-off keying (OOK)-TDM-PON by means of an integrated circuit (IC). The local-area PON exploits the low-cost $\lambda$-tunable vertical cavity surface emitting laser (VCSEL) technology.

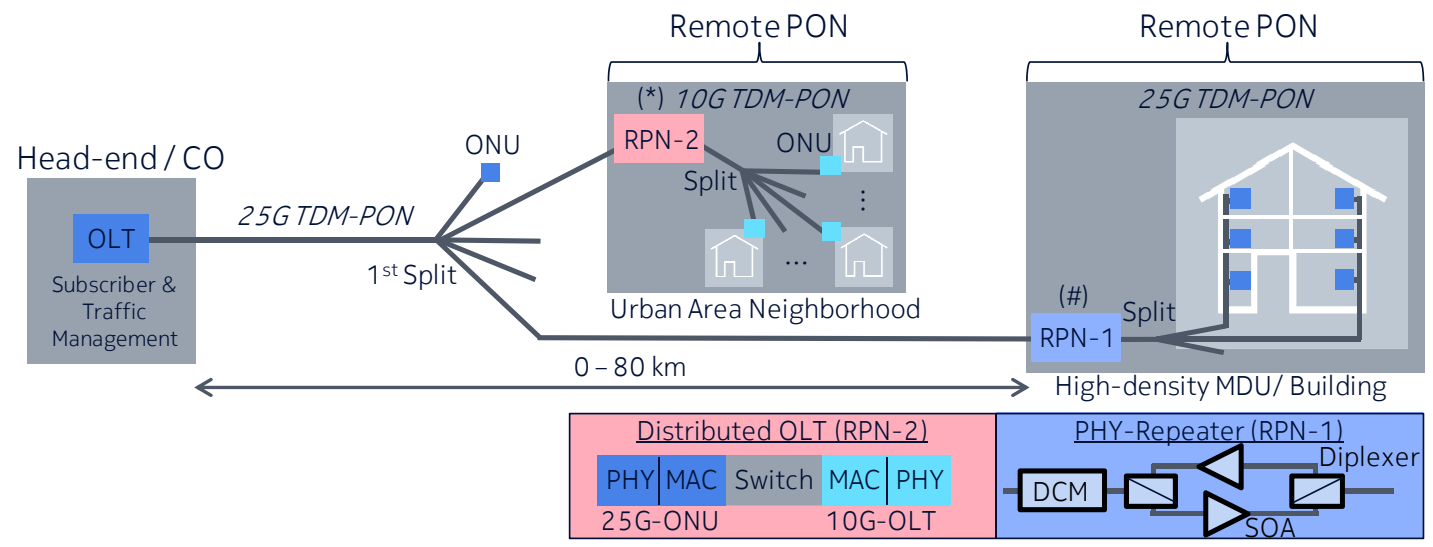

Fig. 1: Remote-PON architecture to increase split and reach in MSO networks. Different remote-PON nodes (RPN) can be established: RPN-1: an extender and RPN-2: a distributed OLT with PHY and MAC functionality while traffic and subscriber management is head-end centralized. 


\section{Network architectures of remote PONs and experimental setup for concept validation}

The deployment of remote-PON architectures can be beneficial for MSO to satisfy their needs for longer fiber lengths and higher number of customers per PON. Fig. 1 shows an architecture in which a $25 \mathrm{Gbit} / \mathrm{s}$ TDM-PON OLT located in the head-end is used to service a number of remote-PON networks. Two applications for distant remote PONs are selected: (\#) a high-density multi-dwelling unit (MDU) and (*) a dense local urban neighborhood.

Solution (\#) can provide a long fiber distance and an adequate split ratio using RPN-1. This node is an extender comprising a dispersion compensation module (DCM), upstream (US) / downstream (DS) diplexer filters and SOAs.

Solution (*) can provide a very high density aggregation of subscribers using RPN-2. This node is a distributed OLT realizing the aggregation of multiple local area lower speed PONs to a higher speed trunk PON. This way, lower cost equipment, e.g. VCSEL-based transmitters, can be leveraged in the remote nodes and networks. The distributed OLT comprises the $25 \mathrm{Gbit} / \mathrm{s}$ optical network unit (ONU) pluggable including physical (PHY) and media access control (MAC) functions, a layer 2 switch and a $10 \mathrm{Gbit} / \mathrm{s}$ OLT pluggable including MAC and PHY-layer for the remote-PON [1]. Functions like traffic and subscriber management are located in the head-end-OLT only. This way, a distributed OLT reduces power consumption, space requirements and cost compared to a fully equipped OLT.

28G-NRZ-OOK-Tx
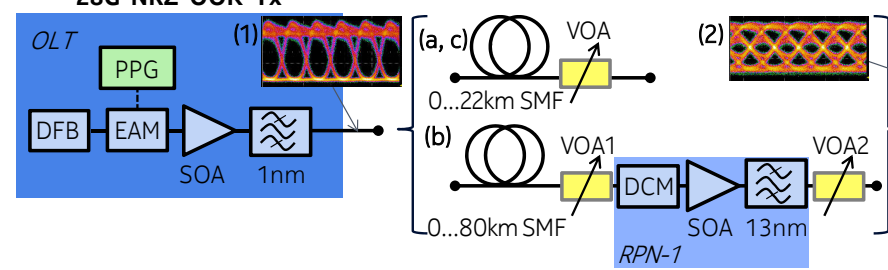

28G-EDB-Rx \& 1:4 Demux

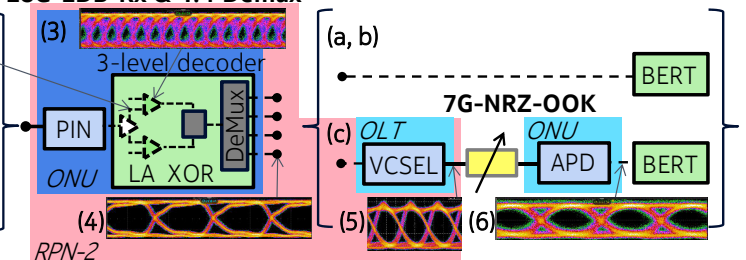

Fig. 2: Setup to validate in real-time both remote-PON concepts: (a) the 28 Gbit/s trunk TDM-PON, (b) RPN-1 and (c) RPN-2 realizations.

The experimental setup used to perform a real-time demonstration of both RPN concepts in downstream direction is shown in Fig. 2. A 28 Gbit/s non-return-to-zero (NRZ)-OOK transmitter (Tx) comprises a distributed feedback laser (DFB) operated at $1538 \mathrm{~nm}$, an electro-absorption modulator (EAM) driven by a pulse pattern generator (PPG) with a pseudo-random binary sequence (PRBS) of $2^{9}-1$, an SOA and a $1 \mathrm{~nm}$ full width at half maximum (FWHM) filter. The achieved OLT-Tx output power is $+10.5 \mathrm{dBm}$ with an extinction ratio (ER) of $8.4 \mathrm{~dB}$. In the following, we describe the different measurement setups for: (a) the characterization of the $28 \mathrm{Gbit} / \mathrm{s}$ EDB-TDM-PON, (b) the RPN-1 realization in the remote-PON concept (\#) and (c) the RPN-2 realization in the remote-PON concept (*).

In case (a), the OLT-Tx signal (eye inset 1) is transmitted over different single mode fiber (SMF) lengths between 0 and $22 \mathrm{~km}$, attenuated by a variable optical attenuator (VOA) mimicking the split losses and subsequently launched into the $28 \mathrm{Gbit} / \mathrm{s}$-capable ONU receiver (Rx). EDB makes use of low-pass filtering and thus, we target the utilization of lower cost $10 \mathrm{Gbit} / \mathrm{s}$ devices at the ONU side. An additional advantage of EDB is the higher chromatic dispersion tolerance compared to NRZ. Thus, in our ONU-Rx implementation, we use a $10 \mathrm{GHz}-\mathrm{PIN}$ photodiode followed by a transimpedance amplifier (TIA) and a 3-level decoder IC chip. The combined frequency response of the PIN/TIA and the 3-level decoder generates a low-pass filtering function for the $28 \mathrm{Gbit} / \mathrm{s}$ NRZ signal resulting in a 3-level EDB signal (eye 2). The decoder IC [4] combines the 3-level signal decoding function and a 1:4 demultiplexer (Demux). It first amplifies the input signal linearly to account for the RF splitter loss. After this splitter the upper and lower eyes are separated and amplified to their logic levels by two limiting amplifiers (LA; eye $3)$. The upper and lower logic signals then enter a high-speed digital XOR gate regenerating the data back into NRZ. Finally, the signal is demultiplexed into 4 outputs (eye 4). A BER tester (BERT) evaluates the performance.

In case (b), the OLT-Tx signal is transmitted over 0, 40 and $80 \mathrm{~km}$ of SMF. The split loss in front of the extender (RPN-1) can be adjusted by the VOA1. The extender-box comprises a DCM (dispersion compensating fiber-DCF) that compensates for chromatic dispersion of the respective SMF length, an SOA with $26.5 \mathrm{~dB}$ smallsignal gain, a $7.5 \mathrm{~dB}$ noise figure and a $-3 \mathrm{~dB}$ saturation input power of $-11 \mathrm{dBm}$, followed by a $13 \mathrm{~nm}$ FWHM US/DS-separation filter. The $28 \mathrm{Gbit} / \mathrm{s}$ signal is adjusted by VOA2 (mimic remote-PON loss) in power before launching into the ONU-Rx and subsequent BER evaluation.

In case (c), the signal path as described in case (a) is reused with few meters of SMF up to the 1:4 demultiplexer output. This $7 \mathrm{Gbit} / \mathrm{s}$ binary electrical output signal is used to directly modulate a $\lambda$-tunable $10 \mathrm{Gbit} / \mathrm{s}$-capable VCSEL [5]. The VCSEL signal output (eye 5) power is $-1.5 \mathrm{dBm}$. The ER is $5.6 \mathrm{~dB}$ at $1543 \mathrm{~nm}$. The remote-PON network loss is set by a VOA and the ONU comprises a $10 \mathrm{Gbit} / \mathrm{s}$ avalanche photodiode (APD)/TIA (eye 6). The performance is again evaluated with a BERT. In duobinary systems, pre-coding of the Tx-data is required to avoid error propagation when using real data. The pre-coder could be omitted in our setup, because the recovered and demultiplexed PRBS is a time-shifted version of the initial PRBS. In our experiments, we realize the distributed OLT on the PHY-layer by de-interleaving the $28 \mathrm{Gbit} / \mathrm{s}$ bit stream into four $7 \mathrm{Gbit} / \mathrm{s}$ bit streams. This way, 
continuous-mode DS traffic is generated for the remote-PON. Here, the 1:4 Demux emulates the conventional packet-based operation of the layer 2 switch in combination with the frame-delimiting functionality of the MAC.

\section{Experimental results}

The power budget offered by the RPN concepts is evaluated using the BER performance. Fig. 3(a) shows the results for the $28 \mathrm{Gbit} / \mathrm{s}$ EDB-TDM-PON, i.e., BER versus PIN photoreceiver input power. A sensitivity of $-17 \mathrm{dBm}$ at a $\mathrm{BER}$ of $1 \mathrm{E}-3$ is obtained. We take this pre-forward-error correction threshold as the reference. Hence, the power budget of this PON is $27.5 \mathrm{~dB}$. The dispersion tolerance of this system is shown in the inset of Fig. 3(a). The power penalty (PP) evaluated at a BER of $1 \mathrm{E}-3$ as a function of the chromatic dispersion is presented. Exemplarily, a chromatic dispersion of $\sim 290 \mathrm{ps} / \mathrm{nm}$ causes $2 \mathrm{~dB}$ PP, offering $18.5 \mathrm{~km}$ differential reach at the Tx wavelength.

The measurement results for the RPN-1 realization employed in the remote-PON concept (\#) are presented in Fig. 3(b). Since the losses (VOA1) in front of the extender-box and the losses (VOA2) behind the extender-box are interrelated, we determine the acceptable losses after the extender as a function of the given losses in front of the extender for $0 \mathrm{~km}(\mathrm{o}), 40 \mathrm{~km}(\mathrm{o})$ and $80 \mathrm{~km}$ (o) of SMF length. The losses are acceptable as long as a BER of 1E-3 is obtained. The results with RPN-1 are compared to the case without amplification (black curve, ${ }^{*}$ ). The low optical signal-to-noise ratio (OSNR) limits the performance for high losses in front of the extender, whereas the thermal noise of the PIN/TIA limits the performance for high losses behind the extender. For the exemplary case of a $40 \mathrm{~km}$ SMF reach, losses of $24 \mathrm{~dB}$ in front of the RPN-1 can be tolerated simultaneously with $25 \mathrm{~dB}$ losses behind the extender, i.e., a $49 \mathrm{~dB}$ total power budget is supported. Assuming C-band operation, fiber loss of $0.25 \mathrm{~dB} / \mathrm{km}$ and a split loss of $3.5 \mathrm{~dB} /$ stage, this budget could be allocated to a 1:16 split ratio of the first splitter, few kilometer SMF reach of the remote PONs and a split ratio of 1:128 each, i.e., 2048 customers per PON. In case of $80 \mathrm{~km}$ reach, the total subscribers per PON can be up to $128(4 \times 32)$. The reduction in power budget for increasing SMF lengths can be attributed to an increased loss of the respective DCF used in RPN-1 to compensate for chromatic dispersion.
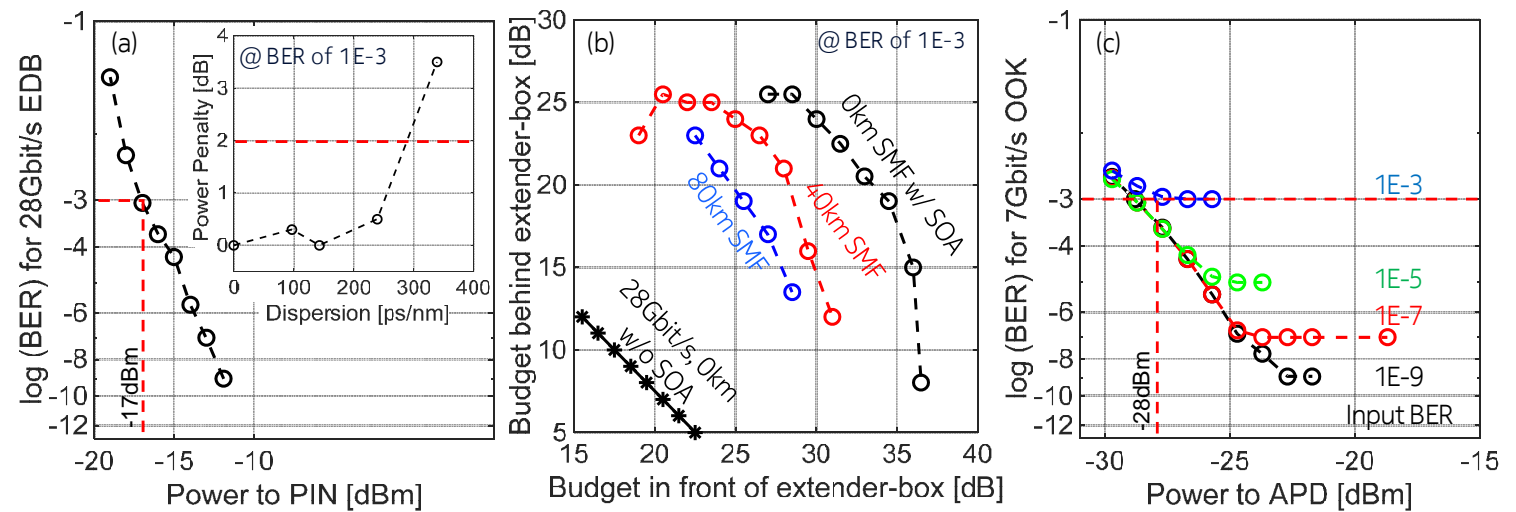

Fig. 3: Results for: (a) 28 Gbit/s EDB-TDM-PON in DS, (b) RPN-1 using an extender and (c) RPN-2 using the PHY-layer of a distributed OLT.

The measurement results for the RPN-2 realization employed in the remote PON concept $(*)$ are presented in Fig. 3(c). The BER for the $7 \mathrm{Gbit} / \mathrm{s}$ NRZ-OOK TDM-remote-PON system as a function of the power to the APD shows that a receiver sensitivity of $-28 \mathrm{dBm}$ at a BER threshold of $1 \mathrm{E}-3$ can be obtained irrespectively of the quality of the input signal arising from the $28 \mathrm{Gbit} / \mathrm{s}$ EDB-TDM-PON (black curve 1E-9 BER input, red curve 1E-7, green curve 1E-5, blue curve 1E-3). The different input BER to the remote-PON stems from the exploitation of the loss budget in the $28 \mathrm{Gbit} / \mathrm{s}$ PON system. A maximum total power budget of $54 \mathrm{~dB}$ is achieved, i.e., $27.5 \mathrm{~dB}$ offered by the $28 \mathrm{Gbit} / \mathrm{s}$ TDM-PON and $26.5 \mathrm{~dB}$ by the $7 \mathrm{Gbit} / \mathrm{s}$ remote TDM-PON. Assuming the given parameters from above, this budget might be allocated to a $40 \mathrm{~km}$ fiber reach and a 1:16 split ratio of the first splitter as well as a short reach of the remote PONs with a split ratio of 1:128 each, i.e., 2048 customers per PON (including the loss of a DCF and the PP induced by the chirp of the VCSEL that is tested to be $<2.5 \mathrm{~dB}$ for $10 \mathrm{~km}$ of SMF).

\section{Conclusion}

We experimentally perform a real-time demonstration of a $28 \mathrm{Gbit} / \mathrm{s}$ EDB-TDM-PON extension by using remotePON nodes either based on SOA or based on a distributed OLT concept. A power budget of about $50 \mathrm{~dB}$ and up to $80 \mathrm{~km}$ fiber reach is shown. As many as 2048 customers per PON can be served at $40 \mathrm{~km}$ reach.

\section{References}

[1] E. Boyd et al., "Remote PON Network Performance", Proc. 2015 Spring Technical Forum, (2015).

[2] IEEE P802.3ca 100G-EPON Task Force, http://www.ieee802.org/3/ca/index.shtml.

[3] V. Houtsma et al., "Demonstration of symmetrical 25 Gbps TDM-PON with 31.5 dB optical power budget using only 10 Gbps optical components", Proc. ECOC, PDP.4.3, (2015).

[4] X. Yin et al., "40-Gb/s TDM-PON Downstream with Low-Cost EML Tx \& 3-Level Detection APD Rx," Proc. OFC, Tu3C.1, (2016).

[5] C. Chase et al., "1550-nm wavelength-tunable HCG VCSELs", Proc. SPIE 8995, High Contrast Metastructures III, 89950A; (2014). 